\title{
The Amsel frustration effect in monkeys'
}

JOHN W. DAVENPORT AND CARL I. THOMPSON

REGIONAL PRIMATE RESEARCH CENTER, UNIVERSITY OF WISCONSIN

\begin{abstract}
Four adolescent stumptail macaques were tested in a discrete-trials retractable-lever analogue of the double runway commonly used to study frustration effects in rats. Each trial consisted of fixed-ratio responding on a left lever followed by an identical contingency on a right lever. In agreement with runway data in rats, right-lever responses were faster following nonreinforcement than following reinforcement of left-lever responses.
\end{abstract}

\section{Problem}

Although the instrumental learning research of Amsel (1962) and others on frustration has developed into one of the major lines of inquiry in learning research, nearly all of this work has been conducted with rats trained in a double runway. In the present study, we attempted to demonstrate the frustration effect (FE) in monkeys using procedures similar to those in the double-runway studies by Amsel \& Roussel (1952) and Wagner (1959). Since the use of an actual runway for monkeys presented practical difficulties, an operant analogue of the double runway was employed.

Using a standard monkey test chamber equipped with two retractable levers, we devised a schedule which may be designated "discrete-trials fixed-ratio (left) fixedratio (right)," or $\mathrm{DT}-\mathrm{FR}_{\mathrm{L}} \mathrm{FR}_{\mathrm{R}}$. In this schedule trials were given in which Ss completed a fixed number of responses on the left lever followed by an equal number of responses on the right lever. The first half of each trial ended with either reward ( $R$ trial) or nonreward (F trial), and the left and right "halves" of a trial were separated by a mid-trial interval (MTI) during which both levers were retracted. With appropriate cues added, the period of responding on the left lever was considered analogous to the "Alley 1" portion of double-runway trials, the right-lever responding to "Alley 2" responding, and the MTI to "mid-box confinement time". This schedule is a simple extension of the discrete-trials single-FR runway analogue used by Karsh (1964) and Porter and Hug (1965).

\section{Method}

Subjects.

The Ss were four adolescent females of the species Macaca speciosa which had previous training on various intermittent reinforcement schedules in free-operant conditioning and in discrimination reversal learning in a $\mathrm{Y}$-maze.

Apparatus.

The apparatus was a Lehigh Valley 1317 operant test chamber equipped with left and right retractable levers, 3-color cue lights above each lever, a set of house lights, and a Davis pellet feeder. The feeder dispensed $45-$ or $97-\mathrm{mg}$ sucrose pellets into a food cup located 3 in below a point midway between the levers, which were 14 in apart. All functions of the test chamber were automatically programmed and recorded.

Trial Procedure.

Training began with the basic schedule used throughout the experiment-discrete trials in which $S$ completed a fixed number of responses on the left lever followed by an equal number on the right lever. The left lever was inserted into the chamber coincident with the onset of the house lights and a green flashing (10/sec.) left cue light. The latter signal remained on throughout the MTI, during which the left lever was retracted and $S$ was required to wait for insertion of the right lever. The second half of each trial started with the termination of the left cue light, the onset of a white flashing (also 10/sec.) right cue light, and the insertion of the right lever. Five sec. after the final response to the right lever and its retraction, the trial ended with the cessation of both the right cue light and house lights, plunging $S$ into an intertrial period of total darkness which averaged $30 \mathrm{sec}$.

Response measures taken throughout the experiment were the latency of the first lever-press and the time to complete the ratio (time from first to last response), in each half of a trial. These measures were converted to "starting speed" and "ratio speed" measures, respectively, by a reciprocal (x 100) transformation. Phase 1.

In the first 10 sessions (days), each $\mathrm{S}$ received 300 trials, 30 per session, with each lever on a 10:1 fixed ratio (DT-FR $\mathrm{L}^{10-\mathrm{FR}_{\mathrm{R}} 10}$ schedule), a 45-mg pellet reinforcement for the 10th response on each lever, and a 10-sec. MTI.

\section{Phase 2.}

Beginning with the 11th session, reinforcement for completing the 10:1 ratio on the left lever was omitted on a random half of the trials, providing a comparison of right-lever measures for equal numbers of $R$ and $F$ trials. Since it appeared that ceiling effects were seriously limiting the degree of difference between R-trial and F-trial speeds, the fixed ratio was permanently increased to 20:1 after the 13th session. Other conditions were altered, one at a time, until arbitrarilydesignated "standard" conditions were established in sessions 22-25. These standard conditions consisted of a larger-size sucrose pellet $(97 \mathrm{mg})$, a shortened MTI (5 sec.), an intertrial interval averaging 30 sec., and a daily mixture of $15 \quad R$ and $15 \mathrm{~F}$ trials on the 
DT-FR ${ }_{L}$ 20-FR $R_{R} 20$ schedule. Phase 2 ended with four sessions (26-29) in which the click of an empty pellet dispenser was presented at the end of left-lever responding on $\mathrm{F}$ trials.

Phase 3.

In order to gain a clear picture of the $\mathrm{FE}$ in relation to consistently reinforced baseline behavior, phase 3 was devoted to: daily alternation (sessions 30-37) of standard mixed-R-F sessions and sessions in which all 30 trials were $\mathrm{R}$ trials; and four additional 30-trial sessions $(38-41)$ in which five selected trials within the middle 10-trial block were $F$ trials. In the last session of this phase, the five $\mathrm{F}$ trials were consecutive.

\section{Results and Discussion}

The FE, defined as faster right-lever responding on $\mathrm{F}$ than on $\mathrm{R}$ trials, appeared within the first two sessions of phase 2, but a sufficiently clear demonstration of it was not evident until the portion of phase 2 that is depicted in Fig. 1, which also shows all of phase 3 . The points plotted in this figure are based on all 30 daily trials except for the last four sessions, for which only the middle 10-trial block is represented. "Mean median" refers both to the averaging of Ss' 5-trial median speeds within sessions and to the

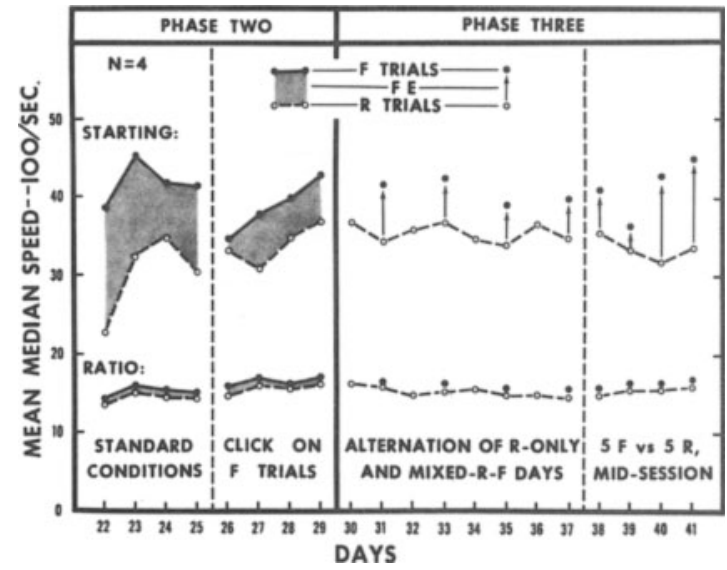

Fig. 1. Performance on the right (second-half) lever in the last eight sessions of phase 2 and all phase 3 sessions for all four Ss combined. averaging of daily individual scores over all Ss. These group data are representative of individual $\mathrm{Ss}$ ' in that all four $\mathrm{Ss}$ exhibited the $\mathrm{FE}$ in nearly every session that is portrayed in the figure, but they conceal a substantial divergence among Ss in its extent.

Figure 1 emphasizes the regularity with which the FE appeared, the fact that it was more clearly seen with the starting than with the ratio speed measure, and (phase 3 ) that its primary form was a speed-up on F trials, as obtained by Amsel \& Roussel (1952) and Wagner (1959), rather than a slowing-down on $\mathrm{R}$ trials. We interpret the temporary reduction in the $\mathrm{FE}$ at session 26 as reflecting "pellet-hunting" behavior elicited by the empty feeder click presented on $F$ trials. No consistent difference was observed in leftlever responding between $\mathrm{R}$ and $\mathrm{F}$ trials, nor between trials immediately following $\mathrm{R}$ trials and those following $F$ trials, in any phase of the experiment.

In summary the experiment demonstrated effects of mid-trial nonreinforcement which closely resembled those seen in most recent frustration studies with rats. The way now seems clear to continue this fruitful line of inquiry with automated discrete-trials methods which are applicable to a much wider range of species.

\section{References}

Amsel, A. Frustrative nonreward in partial reinforcement and discrimination learning: Some recent history and a theoretical extension. Psychol. Rev., 1962, 69, 306-328.

Amsel, A., \& Roussel, J. Motivational properties of frustration: I. Effect on a running response of the addition of frustration to the motivational complex. J. exp. Psychol., 1952, 43, 363-368.

Karsh, E. B. Punishment: trial spacing and shock intensity as determinants of behavior in a discrete operant situation. J. comp. physiol. Psychol., 1964, 58, 299-302.

Porter, J. J., \& Hug, J. J. Discrete-trial bar-pressing performance as a function of length of response chain, number of reinforcements, and percentage reward. Psychol. Rep., 1965, 16, 11091116.

Wagner, A. R. The role of reinforcement and nonreinforcement in an "apparent frustration effect." J. exp. Psychol., 1959, 57, 130136.

\section{Note}

1. This research was supported by grants FR-00167 and MH- 4528 from the National Institutes of Health. We thank Constance Poe for assistance in data tabulation. 